\title{
DILATION THEOREMS FOR COMPLETELY POSITIVE MAPS AND MAP-VALUED MEASURES
}

\author{
EWA HENSZ-CHA̧DZYŃSKA, RYSZARD JAJTE and ADAM PASZKIEWICZ \\ Faculty of Mathematics, Eódź University \\ ul. Banacha 22, 90-238 Eódź, Poland \\ E-mail:ewahensz@math.uni.lodz.pl,rjajte@math.uni.lodz.pl,adampasz@math.uni.lodz.pl
}

\begin{abstract}
The Stinespring theorem is reformulated in terms of conditional expectations in a von Neumann algebra. A generalisation for map-valued measures is obtained.

1. Introduction. Traditionally, each dilation theorem is obtained by a construction of a 'huge' (Hilbert) space $\mathcal{H}$ containing a given space $H$ in the following manner. A system $\psi(\cdot)$ of operators in $H$ or transformations of an algebra acting in $H$ can be represented in the form

$$
\psi(\cdot)=P_{H} \Phi(\cdot) P_{H \mid H}
$$

where $\Phi(\cdot)$ is more regular than $\psi(\cdot)$. Throughout, $P_{H}$ denotes the orthogonal projection of $\mathcal{H}$ onto $H$.

The most impressive results in this theory are effects of sophisticated indexing of linear bases of $\mathcal{H}$ and a 'magic touch' of scalar product. Theorems of B. Sz.-Nagy [9] and K.R. Parthasarathy [5] are excellent examples of such approach.

Dealing with operator algebras it seems to be most natural and physically meaningful to use the conditional expectation $\mathbb{E}[7, \mathrm{p} .116]$ instead of $P_{H}(\cdot) P_{H}$ (cf L. Accardi, M. Ohya [1]).

In the paper we follow both ideas. Roughly speaking we represent a completely positive map-valued measure via the following dilation. Namely, any completely positive map turns into multiplication by a projection in such a way that the map-valued measure is 'dilated' to a spectral measure (Section 2).
\end{abstract}

1991 Mathematics Subject Classification: 46L50, 28 B05.

Key words and phrases: dilation, von Neumann algebra, completely positive map, map-valued measure.

Research supported by KBN grant 2 P 03A 04808.

The paper is in final form and no version of it will be published elsewhere. 
The outstanding theorem of Stinespring [6] gives the dilation of a completely positive map $\psi$ in a $C^{*}$-algebra to its $*$-representation $\Phi$ via formula (1.1). Passing to a $W^{*}$-algebra $\mathcal{M}$ Stinespring's theorem can be formulated using a normal conditional expectation $\mathbb{E}$ from a 'huge' algebra $\mathcal{N}$ onto $\mathcal{M}$ instead of $P_{H}(\cdot) P_{H}$. Such a new version of Stinespring's result will be proved in Section 3 together with a dilation theorem for positive map-valued measures.

Section 4 is devoted to a short comparison of the results just mentioned with the previous ones concerning commutative $W^{*}$-algebras.

2. Dilation of completely positive map-valuded measure. Let $\mathcal{M}$ be a von Neumann algebra of operators acting in a Hilbert space $H$. By $C P(\mathcal{M})$ we shall denote the set of completely positive linear maps in $\mathcal{M}$. Let $(X, \Sigma)$ be a measurable space and $Q: \Sigma \rightarrow C P(\mathcal{M})$ be a $\sigma$-additive operator-valued measure (i.e. $\Sigma \ni \Delta \mapsto Q(\Delta) x$ is $\sigma$-additive in the ultra weak topology in $\mathcal{M}$ for each $x \in \mathcal{M})$ with $Q(X) 1=1$.

THEOREM 2.1. There exist a Hilbert space $\mathcal{H}$, a natural linear injection $V: H \rightarrow \mathcal{H}$, $a *$-representation $\Phi$ of the algebra $\mathcal{M}$ in $\mathcal{H}$, a $\sigma$-additive vector measure $e: \Sigma \rightarrow$ Proj $\mathcal{H}$, such that

$$
Q(\Delta) x=V^{*} e(\Delta) \Phi(x) V, \quad x \in \mathcal{M}, \quad \Delta \in \Sigma .
$$

Moreover, $e(\Delta)$ is a central projection in $(\Phi(\mathcal{M}) \cup e(\Sigma))^{\prime \prime}$.

Proof. Let us consider the algebraic tensor product of vector spaces

$$
\mathcal{H}_{0}=\mathcal{M} \otimes H \otimes S F(X, \Sigma)
$$

where $S F(X, \Sigma)$ denotes the vector space of simple functions on $(X, \Sigma)$.

Let us extend the measure $Q$ from $\Sigma$ to a linear mapping on $S F(X, \Sigma)$ putting

$$
Q(f)=\sum_{\kappa=1}^{k} c_{\kappa} Q\left(\Delta_{\kappa}\right) \quad \text { for } \quad f=\sum_{\kappa=1}^{k} c_{\kappa} 1_{\Delta_{\kappa}}
$$

where $\Delta_{\kappa} \in \Sigma, \kappa=1, \ldots, k$.

In the sequel we shall briefly write $\Delta$ instead of $1_{\Delta}, \Delta \in \Sigma$. Notice that $\mathcal{H}_{0}$ is formed by elements of the form

$$
\xi=\sum_{i=1}^{n} x_{i} \otimes h_{i} \otimes \Delta_{i}
$$

where $x_{i} \in \mathcal{M}, h_{i} \in H, \Delta_{i} \in \Sigma, i=1, \ldots, n, n=1,2, \ldots$.

In the space $\mathcal{H}_{0}$ we can define a sesquilinear form $\langle\cdot, \cdot\rangle$ by

$$
\langle\xi, \eta\rangle=\sum_{i=1}^{n} \sum_{j=1}^{m}\left(Q\left(\Delta_{i} \cap \Gamma_{j}\right)\left(y_{j}^{*} x_{i}\right) h_{i}, g_{j}\right)
$$

for

$$
\xi=\sum_{i=1}^{n} x_{i} \otimes h_{i} \otimes \Delta_{i} \quad \text { and } \quad \eta=\sum_{j=1}^{m} y_{j} \otimes g_{j} \otimes \Gamma_{j} .
$$

The symbol $(\cdot, \cdot)$ denotes here the inner product in $H$. We shall show that $\langle\cdot, \cdot\rangle$ is positive. Indeed, for $\xi$ of form (2.2) we consider the partition $\left\{\sigma_{1}, \ldots, \sigma_{k}\right\}$ of $\bigcup_{i=1}^{n} \Delta_{i}$ given by 
$\Delta_{i}, \ldots, \Delta_{n}$. Putting $\varepsilon_{s}^{i}=1$ when $\sigma_{s} \subset \Delta_{i}$ and $\varepsilon_{s}^{i}=0$ when $\sigma_{s} \cap \Delta_{i}=\emptyset$ we can write

$$
\begin{aligned}
\langle\xi, \xi\rangle & =\sum_{i, j=1}^{n}\left(Q\left(\Delta_{i} \cap \Delta_{j}\right)\left(x_{j}^{*} x_{i}\right) h_{i}, h_{j}\right)=\sum_{i, j=1}^{n}\left(\left(\sum_{s=1}^{k} \varepsilon_{s}^{i} \varepsilon_{s}^{j} Q\left(\sigma_{s}\right)\right)\left(x_{j}^{*} x_{i}\right) h_{i}, h_{j}\right) \\
& =\sum_{s=1}^{k} \sum_{i, j=1}^{n}\left(Q\left(\sigma_{s}\right)\left(x_{j}^{*} x_{i}\right) h_{i}^{s}, h_{j}^{s}\right)
\end{aligned}
$$

where $h_{i}^{s}=\varepsilon_{s}^{i} h_{i}, i=1, \ldots, n$.

The complete positivity of $Q\left(\sigma_{s}\right)$ gives the inequality

$$
\sum_{i, j=1}^{n}\left(Q\left(\sigma_{s}\right)\left(x_{j}^{*} x_{i}\right) h_{i}^{s}, h_{j}^{s}\right) \geq 0, \quad s=1, \ldots, k,
$$

thus $\langle\xi, \xi\rangle \geq 0$. Let us denote $\|\xi\|_{0}=\sqrt{\langle\xi, \xi\rangle}$ and put $\mathcal{H}_{1}=\mathcal{H}_{0} / N$ where $N=\left\{\xi \in \mathcal{H}_{0}\right.$ : $\left.\|\xi\|_{0}=0\right\}$. Finally, let us set $\left.\mathcal{H}=\overline{\mathcal{H}}_{1} \cdot \cdot \cdot \cdot\right\rangle$.

We define $V: H \rightarrow \mathcal{H}$ by putting $V h=[1 \otimes h \otimes X]$ for $h \in H$. Then

$$
\langle V h, V h\rangle=((Q(X) 1) h, h)=(h, h)
$$

so $V$ is an isometry.

Now let us construct a $*$-representation $\Phi$ of the algebra $\mathcal{M}$ in $\mathcal{H}$. Namely, for $x \in \mathcal{M}$ let us set

$$
\Phi(x):[y \otimes h \otimes \Delta] \mapsto[x y \otimes h \otimes \Delta]
$$

where $y \in \mathcal{M}, h \in H, \Delta \in \Sigma$. $\Phi(x)$ is well defined. Indeed, we prove the following inequality

$$
\left\|\sum_{i=1}^{n} x y_{i} \otimes h_{i} \otimes \Delta_{i}\right\|_{0} \leq\|x\| \cdot\left\|\sum_{i=1}^{n} y_{i} \otimes h_{i} \otimes \Delta_{i}\right\|_{0}
$$

for $y_{i} \in \mathcal{M}, h_{i} \in H, \Delta_{i} \in \Sigma, i=1, \ldots, n, n=1,2, \ldots$ As above, we can write

$$
\begin{aligned}
\left\|\sum_{i=1}^{n} y_{i} \otimes h_{i} \otimes \Delta_{i}\right\|_{0}^{2} & =\sum_{s=1}^{k} \sum_{i, j=1}^{n}\left(Q\left(\sigma_{s}\right)\left(y_{j}^{*} y_{i}\right) h_{i}^{s}, h_{j}^{s}\right), \\
\left\|\sum_{i=1}^{n} x y_{i} \otimes h_{i} \otimes \Delta_{i}\right\|_{0}^{2} & =\sum_{s=1}^{k} \sum_{i, j=1}^{n}\left(Q\left(\sigma_{s}\right)\left(y_{j}^{*} x^{*} x y_{i}\right) h_{i}^{s}, h_{j}^{s}\right) .
\end{aligned}
$$

For a linear map $\alpha: \mathcal{M} \rightarrow \mathcal{M}$ let us denote by $\alpha^{(n)}$ the map $\alpha^{(n)}: \operatorname{Mat}_{n}(\mathcal{M}) \rightarrow$ $\operatorname{Mat}_{n}(\mathcal{M})$ given by the formula

$$
\alpha^{(n)}\left(\left[z_{i, j}\right]\right)=\left[\alpha\left(z_{i, j}\right)\right]
$$

where $\left[z_{i, j}\right]_{i, j \leq n} \in \operatorname{Mat}_{n}(\mathcal{M})$. $\operatorname{Mat}_{n}(\mathcal{M})$ denotes here the $C^{*}$-algebra of all $n \times n$ matrices $\left[z_{i, j}\right]_{i, j \leq n}$ with entries $z_{i, j}$ in $\mathcal{M}$.

Now, we follow Takesaki $[10$, p. 196]. The Schwarz inequality for operators, by the complete positivity of $Q\left(\sigma_{s}\right)$, gives

$$
Q\left(\sigma_{s}\right)^{(n)}\left(\widetilde{y}^{*} \widetilde{x}^{*} \widetilde{x} \widetilde{y}\right) \leq\|\widetilde{x}\|^{2} Q\left(\sigma_{s}\right)^{(n)}\left(\widetilde{y}^{*} \widetilde{y}\right)
$$


for each $\widetilde{x}, \widetilde{y} \in \operatorname{Mat}_{n}(\mathcal{M})$. Setting $\widetilde{x}=\left[\delta_{i, j} x\right], \widetilde{y}=\left[\delta_{1, i} y_{i}\right]$ we get $\widetilde{y}^{*} \widetilde{x}^{*} \widetilde{x} \widetilde{y}=\left[y_{i}^{*} x^{*} x y_{j}\right]$, $\widetilde{y}^{*} \widetilde{y}=\left[y_{i}^{*} y_{j}\right]$. Thus, by $(2.5)$ and $\|\widetilde{x}\|=\|x\|$, we have

$$
\left[Q\left(\sigma_{s}\right)\left(y_{i}^{*} x^{*} x y_{j}\right)\right] \leq\|x\|^{2}\left[Q\left(\sigma_{s}\right)\left(y_{i}^{*} y_{j}\right)\right] .
$$

Hence

$$
\sum_{i, j=1}^{n}\left(Q\left(\sigma_{s}\right)\left(y_{i}^{*} x^{*} x y_{j}\right) h_{j}^{s}, h_{i}^{s}\right) \leq\|x\|^{2} \sum_{i, j=1}^{n}\left(Q\left(\sigma_{s}\right)\left(y_{i}^{*} y_{j}\right) h_{j}^{s}, h_{i}^{s}\right) .
$$

Finally, by (2.4), we get (2.3). Then $\left\|\sum_{i=1}^{n} y_{i} \otimes h_{i} \otimes \Delta_{i}\right\|_{0}=0$ implies $\| \sum_{i=1}^{n} x y_{i} \otimes h_{i} \otimes$ $\Delta_{i} \|_{0}=0$ and $\Phi(x)$ is well defined. Obviously by $(2.3), \Phi(x): \mathcal{H} \rightarrow \mathcal{H}$ is a linear bounded operator in $B(\mathcal{H})$. It is easy to check that $\Phi: \mathcal{M} \rightarrow B(\mathcal{H})$ is a $*$-representation $\mathcal{M}$ in $\mathcal{H}$.

Now for $\Delta \in \Sigma$ we define $e(\Delta): \mathcal{H} \rightarrow \mathcal{H}$ putting

$$
e(\Delta):\left[y \otimes h \otimes \Delta^{\prime}\right] \mapsto\left[y \otimes h \otimes\left(\Delta \cap \Delta^{\prime}\right)\right]
$$

where $y \in \mathcal{M}, h \in H, \Delta^{\prime} \in \Sigma$. The operator $e(\Delta)$ is well defined because $\| \sum_{i=1}^{n} y_{i} \otimes h_{i} \otimes$ $\Delta_{i} \|_{0}=0$ implies $\left\|\sum_{i=1}^{n} y_{i} \otimes h_{i} \otimes\left(\Delta \cap \Delta_{i}\right)\right\|_{0}=0$. Indeed, let $\left\{\sigma_{1}, \ldots, \sigma_{k}\right\}$ be a partition of $\bigcup_{i=1}^{n} \Delta_{i}$ given by $\Delta, \Delta_{1}, \ldots, \Delta_{k}$. Let us put $\varepsilon_{s}^{i}=1$ when $\sigma_{s} \subset \Delta_{i}$ and $\varepsilon_{s}^{i}=0$ when $\sigma_{s} \cap \Delta_{i}=\emptyset$. Similarly, let $\varepsilon_{s}=1$ when $\sigma_{s} \subset \Delta$ and $\varepsilon_{s}=0$ when $\sigma_{s} \cap \Delta=\emptyset$. Then

$$
\begin{aligned}
\| \sum_{i=1}^{n}\left(y_{i} \otimes h_{i} \otimes\left(\Delta \cap \Delta_{i}\right) \|_{0}^{2}\right. & \left.=\sum_{s=1}^{k} \varepsilon_{s} \sum_{i, j=1}^{n} \varepsilon_{s}^{i} \varepsilon_{s}^{j} Q\left(\sigma_{s}\right)\left(y_{j}^{*} y_{i}\right) h_{i}, h_{j}\right) \\
& \leq \sum_{s=1}^{k} \sum_{i, j=1}^{n} \varepsilon_{s}^{i} \varepsilon_{s}^{j}\left(Q\left(\sigma_{s}\right)\left(y_{j}^{*} y_{i}\right) h_{i}, h_{j}\right)=\left\|\sum_{i=1}^{n} y_{i} \otimes h_{i} \otimes \Delta_{i}\right\|_{0}^{2}
\end{aligned}
$$

because, by the complete positivity of $Q\left(\sigma_{s}\right)$, we have

$$
\sum_{i, j=1}^{n} \varepsilon_{s}^{i} \varepsilon_{s}^{j}\left(Q\left(\sigma_{s}\right)\left(y_{j}^{*} y_{i}\right) h_{i}, h_{j}\right) \geq 0 .
$$

Obviously, $e(\Delta)$ is an orthogonal projection in $\mathcal{H}$. Moreover, for $x \in \mathcal{M}$ and $\Delta \in \Sigma$ we have

$$
\Phi(x) e(\Delta)\left[y \otimes h \otimes \Delta^{\prime}\right]=e(\Delta) \Phi(x)\left[y \otimes h \otimes \Delta^{\prime}\right]
$$

where $y \in \mathcal{M}, h \in H, \Delta^{\prime} \in \Sigma$, so $e(\Delta)$ is a central projection in the algebra $\left(\Phi(\mathcal{M} \cup e(\Sigma))^{\prime \prime}\right.$.

Finally, for all $h, g \in H, x \in \mathcal{M}$ and $\Delta \in \Sigma$

$$
\left(V^{*} e(\Delta) \Phi(x) V h, g\right)=\langle e(\Delta) \Phi(x) V h, V g\rangle=\langle x \otimes h \otimes \Delta, 1 \otimes g \otimes X\rangle=((Q(\Delta) x) h, g),
$$

so formula (2.1) holds.

3. Dilations via conditional expectations. At the very beginning dilation theory was motivated by physical applications. In particular, the classical Naimark theorem gives a construction of a good self-adjoint quantum observable expressed by its spectral measure beyond the Hilbert space $H$ in which acts a 'candidate' for physical observable being only an unbounded symmetric operator (see [9] for precise explanation). On the other hand, passing from a given operator algebra to a bigger one, physically means passing from a given system to a bigger one. That is why general ideas of dilation theory can be interpreted as follows. Enlarging a Hilbert space we usually pass to a new (better) model 
of the same physical system whereas the construction of a dilation in a bigger algebra means passing to a bigger system enjoying more regular evolution ([3], [2]).

In particular, the physical meaning of Stinespring's theorem can be enriched if we express the dilation in terms of the conditional expectation in the enlarged algebra. Such a construction, with consequences for map-valued measures, will be done in this section.

It turns out that some natural properties of an equivalence relation in the lattice of projections are crucial.

A basic tool is the comparison theorem for projections ([8], Thm. 4.6).

Theorem 3.1. For any $p, q \in \operatorname{Proj} \mathcal{N}$, there exists a projection $e \in \mathcal{N} \cap \mathcal{N}^{\prime}$ such that $p e \succcurlyeq q e$ and $p(1-e) \preccurlyeq q(1-e)$.

Clearly, $p \preccurlyeq q$ means $u u^{*}=p, u^{*} u \leq q$ for some partial isometry $u \in \mathcal{N}$.

The following consequence of the above theorem will be used.

Proposition 3.2. Let $\mathcal{N}$ be a von Neumann algebra and let $p$ be a projection in $\mathcal{N}$ with the central support $z(p)=1$. There exists a system of mutually orthogonal projections $\left(p_{i} ; i<k_{0}\right)$ in $\operatorname{Proj} \mathcal{N}, k_{0}$ being an ordinal number, such that $p_{i} \preccurlyeq p, \sum_{i<k_{0}} p_{i}=1$, and $p_{1}=p$.

Proof. We use the transfinite induction, treating $1,2, \ldots$ as ordinals. Denote $e_{1}=0$, $p_{1}=p$. Assume that, for some ordinals $k$ and for any $i<k$, projections $e_{i}, p_{i} \in \operatorname{Proj} \mathcal{N}$ satisfying the conditions

$$
\begin{aligned}
& e_{i} \in \mathcal{N}^{\prime}, \\
& \left(e_{i} ; i<k\right) \text { are mutually orthogonal, } \\
& \left(p_{i} ; i<k\right) \text { are mutually orthogonal, } \\
& \sum_{j \leq i} p_{j} \geq \sum_{j \leq i} e_{j}, \\
& p_{i} \preccurlyeq p
\end{aligned}
$$

have already been defined. If $\sum_{i<k} p_{i}=1$, the construction is complete with $k_{0}=k$.

If not, we consider separately the following two cases.

Case $1^{0}$. Assume that

$$
\left(\sum_{j<k} e_{j}\right)^{\perp} p \preccurlyeq\left(\sum_{j<k} e_{j}\right)^{\perp}\left(\sum_{j<k} p_{j}\right)^{\perp} .
$$

Then it is enough to put $e_{k}=0, p_{k}$ an arbitrary projection in $\mathcal{N}$ satisfying

$$
p_{k} \sim\left(\sum_{j<k} e_{j}\right)^{\perp} p, \quad p_{k} \leq\left(\sum_{j<k} e_{j}\right)^{\perp}\left(\sum_{j<k} p_{j}\right)^{\perp}
$$

(clearly, $p \sim q$ means $p=u^{*} u, q=u u^{*}$, for some $u \in \mathcal{N}$ ).

Case $2^{0}$. Assume that $(*)$ does not hold. Then we consider the algebra

$$
\mathcal{M}=\left(\sum_{j<k} e_{j}\right)^{\perp} \mathcal{N}\left(\sum_{j<k} e_{j}\right)^{\perp}=\mathcal{N}\left(\sum_{j<k} e_{j}\right)^{\perp} .
$$

Restricting operators to a subspace $\left(\sum_{j<k} e_{j}\right)^{\perp}(H)$, one can treat $\mathcal{M}$ as a von Neumann algebra with the projections $\widetilde{p}=p\left(\sum_{j<k} e_{j}\right)^{\perp}, \widetilde{p}_{i}=p_{i}\left(\sum_{j<k} e_{j}\right)^{\perp}$. By the comparison 
theorem there exists a central projection in $\mathcal{M}$, say $e_{k}$, satisfying the conditions

$$
\widetilde{p} e_{k} \succcurlyeq\left(\sum_{j<k} \widetilde{p}_{j}\right)^{\perp} e_{k} \text { and } \widetilde{p}\left(1_{\mathcal{M}}-e_{k}\right) \preccurlyeq\left(\sum_{j<k} \widetilde{p}_{j}\right)^{\perp}\left(1_{\mathcal{M}}-e_{k}\right) \text {. }
$$

Since the reduction of $\mathcal{N}$ to $\mathcal{M}$ is done by the central projection $\left(\sum_{j<k} e_{j}\right)^{\perp}, e_{k}$ can be obviously treated as a central projection in $\mathcal{N}$ as well.

Let $\widetilde{p}_{k}$ be an arbitrary projection in $\mathcal{M}$ satisfying

$$
\widetilde{p}_{k} \leq\left(\sum_{j<k} p_{j}\right)^{\perp}\left(1_{\mathcal{M}}-e_{k}\right), \quad \widetilde{p}_{k} \sim p\left(1_{\mathcal{M}}-e_{k}\right) .
$$

We put

$$
p_{k}=\widetilde{p}_{k}+\left(\sum_{j<k} p_{j}\right)^{\perp} e_{k}
$$

Obviously, we can treat $p_{k}$ as a projection in $\mathcal{N}$. All conditions (3.1) are now satisfied for $k+1$ (instead for $k$ ).

Clearly, $\sum_{i<k} p_{i}=1$ necessarily for some ordinal $k$ (since $\operatorname{dim} H$ is a fixed cardinal).

We shall need the following consequences of Proposition 3.2.

Lemma 3.3. Let $\mathcal{M}$ and $\mathcal{N}$ be von Neumann algebras acting in Hilbert spaces $H$ and $\mathcal{H}$, respectively, with $H \subset \mathcal{H}$. Denote by $P_{H}$ the orthogonal projection from $\mathcal{H}$ onto $H$. Assume that

$$
P_{H}^{*} \mathcal{M} P_{H} \subset \mathcal{N}, \text { the central support } z\left(P_{H}\right)=1 \text {. }
$$

Then there exists an isometric injection $v: \mathcal{H} \rightarrow H \otimes K$, for some Hilbert space $K$ such that

$$
\begin{gathered}
v \mathcal{N} v^{*} \subset \mathcal{M} \otimes B(K), \\
v \zeta=\zeta \otimes \eta_{1}, \quad \zeta \in H, \quad \text { for some } \eta_{1} \in K .
\end{gathered}
$$

Proof. Keeping the notation of Proposition 3.2, with $p=P_{H} \subset \mathcal{N}$, let us fix a Hilbert space $K$ with an orthogonal basis $\left(\eta_{j}, j<k_{0}\right)$. As $p_{i} \preccurlyeq p$, we can use projections $r_{i} \leq p$ satisfying $p_{i}=w_{i}^{*} w_{i}, r_{i}=w_{i} w_{i}^{*}$ for some partial isometries $w_{i} \in \mathcal{N}, i<k_{0}$. Obviously, we can assume that $w_{1}=p$.

Let us take $v_{i} \zeta=w_{i} \zeta \otimes \eta_{i}, i<k_{0}$, for $\zeta \in H$. Then we get an isometry

$$
v=\sum_{i<k_{0}} v_{i}, \quad v: \mathcal{H} \rightarrow H \otimes K .
$$

Formula (3.4) is obvious. It remains to show (3.3) or, equivalently, $\mathcal{N} \subset v^{*} \mathcal{M} \otimes B(K) v$. This can be checked by the commutant technique as follows.

We have

$$
p_{i} \mathcal{N} p_{i} \cup\left\{w_{i}^{*}, w_{i}\right\} \subset v^{*} \mathcal{M} \otimes B(K) v, \quad i<k_{0} .
$$

Indeed,

$$
\begin{aligned}
p_{i} \mathcal{N} p_{i} & =v^{*}\left(r_{i} \mathcal{M} r_{i} \otimes\left\langle\cdot, \eta_{i}\right\rangle \eta_{i}\right) v, \\
w_{i} & =v^{*}\left(r_{i} \otimes\left\langle\cdot, \eta_{i}\right\rangle \eta_{1}\right) v, \\
w_{i}^{*} & =v^{*}\left(r_{i} \otimes\left\langle\cdot, \eta_{1}\right\rangle \eta_{i}\right) v .
\end{aligned}
$$


For example we check the first equality. Obviously $p_{i} \mathcal{N} p_{i}=w_{i}^{*} \mathcal{N} w_{i}=w_{i}^{*} \mathcal{M} w_{i}$, and for any $x \in \mathcal{M}, \zeta \in \mathcal{H}$, denoting $\zeta_{j}=p_{j} \zeta, j<k_{0}$, we have

and

$$
v \zeta=\sum_{j<k_{0}} w_{j} \zeta_{j} \otimes \eta_{j}
$$

$$
\begin{aligned}
v^{*}\left(r_{i} x r_{i} \otimes\left\langle\cdot, \eta_{i}\right\rangle \eta_{i}\right) v \zeta & \left.=v^{*}\left(r_{i} x w_{i} \zeta_{i} \otimes \eta_{i}\right)=\left(w_{i} \cdot \otimes \eta\right) i\right)^{*}\left(r_{i} x w_{i} \zeta_{i} \otimes \eta_{i}\right) \\
& =w_{i}^{*} x w_{i} \zeta_{i}=w_{i}^{*} x w_{i} \zeta .
\end{aligned}
$$

On the other hand, we have

$$
\left(\bigcup_{i<k_{0}} p_{i} \mathcal{N} p_{i} \cup\left\{w_{i}, w_{i}^{*}\right\}\right)^{\prime}=\mathcal{N}^{\prime} .
$$

The inclusion "つ" is obvious. Conversely, let $y$ commute with all $p_{i} \mathcal{N} p_{i}, w_{i}, w_{i}^{*}$. An arbitrary $z \in \mathcal{N}$ can be represented as $z=\sum_{i, j<k_{0}} p_{i} z p_{j}$. Take $x \in \mathcal{N}$ of the form $x=p_{i} z p_{j}$. We have, since $w_{i} z w_{j}^{*} \in p_{1} \mathcal{N} p_{1}$,

$$
y x=y w_{i}^{*} w_{i} z w_{j}^{*} w_{j}=w_{i}^{*} y\left(w_{i} z w_{j}^{*}\right) w_{j}=w_{i}^{*}\left(w_{i} z w_{j}^{*}\right) y w_{j}=x y .
$$

Taking commutants on both sides of (3.6) and taking into account (3.5), we get (3.3).

Proposition 3.4. For any completely positive map $\alpha$ in a von Neumann algebra $\mathcal{M}$ acting in a Hilbert space $H$ there exists a Hilbert space $K$ and a $*$-representation $\Phi: \mathcal{M} \rightarrow \mathcal{M} \otimes B(K)$ satisfying

$$
\alpha x=\Pi^{*} \Phi(x) \Pi
$$

where, for $\xi \in H, \Pi \xi=\xi \otimes \eta_{1}$ for a fixed vector $\eta_{1} \in K,\left\|\eta_{1}\right\|=1$.

Pr oof. Take any Stinespring triple: $\left(\mathcal{H}, P_{H}, \Psi\right)$ where $\mathcal{H} \supset H, P_{H}$ is an orthogonal projection of $\mathcal{H}$ onto $H$, and $\Psi: \mathcal{M} \rightarrow B(\mathcal{H})$ is a $*$-representation satisfying

$$
\alpha x=\left.P_{H} \Psi(x) P_{H}\right|_{H} .
$$

Denote $\mathcal{N}=(\mathcal{M} \cup \Psi(\mathcal{M}))^{\prime \prime}$ (obviously, we identify $\mathcal{M} \ni x \equiv x P_{H} \in B(\mathcal{H})$ ). According to the Stinespring's construction [6], [10, p. 195] the projection $P_{H}$ has in $\mathcal{N}$ the central support $z\left(P_{H}\right)=1_{\mathcal{N}}$. By Lemma 3.3, there exists a Hilbert space $K$, an isometry $v$ : $\mathcal{H} \rightarrow H \otimes K$ and a vector $\eta_{1} \in K$ satisfying (3.3) and (3.4). We set

$$
\Phi(x)=v \Psi(x) v^{*}, \quad x \in \mathcal{M} .
$$

Then $\Phi$ is a $*$-representation of $\mathcal{M}$ into $\mathcal{M} \otimes B(K)$. Moreover, as $\Pi \xi=\xi \otimes \eta_{1}$ for $\xi \in H$, we have, for any $x \in \mathcal{M}$,

$$
\begin{aligned}
\left(\Pi^{*} \Phi(x) \Pi\right) \xi & =\left(\Pi^{*} v \Psi(x) v^{*}\right)\left(\xi \otimes \eta_{1}\right)=\Pi^{*} v \Psi(x) \xi \\
& =\Pi^{*} v \Psi(x) P_{H} \xi=P_{H} \Psi(x) P_{H} \xi=\alpha(x) \xi
\end{aligned}
$$

(since $\left\langle v^{*}\left(\xi \otimes \eta_{1}\right), \zeta\right\rangle=\langle\xi, \zeta\rangle,\left\langle\Pi^{*} v \rho, \zeta\right\rangle=\left\langle v\left(P_{H} \rho+P_{H}^{\perp} \rho\right), \zeta \otimes \eta_{1}\right\rangle=\left\langle\left(P_{H} \rho\right) \otimes \eta_{1}, \zeta \otimes \eta_{1}\right\rangle=$ $\left\langle P_{H} \rho, \zeta\right\rangle$ for $\zeta \in H, \rho \in \mathcal{H}$, the orthogonality $v P_{H}^{\perp} \rho \perp \zeta \otimes \eta_{1}$ is a consequence of (3.4)).

Now we are in a position to prove dilation theorems in the language of conditional expectations in $W^{*}$-algebras (see [7], Chapter 2 for basic facts).

THEOREM 3.5. For any $W^{*}$-algebra $\mathcal{M}$ and any completely positive map $\alpha$ in $\mathcal{M}$ there exist a $W^{*}$-algebra $\mathcal{N}, \mathcal{N} \supset \mathcal{M}$ (i.e. $\mathcal{M}$ is a $W^{*}$-subalgebra of $\mathcal{N}$ ) and a *-representation 
$\Phi: \mathcal{M} \rightarrow \mathcal{N}$ such that

$$
\alpha x=\mathbb{E}^{\mathcal{M}} \Phi(x), \quad x \in M,
$$

where $\mathbb{E}^{\mathcal{M}}$ is a normal conditional expectation of $\mathcal{N}$ onto $\mathcal{M}$.

Pr o of. We keep the notation of Proposition 3.4. We identify $\mathcal{M}$ with $\mathcal{M} \otimes 1_{K}$ by a natural isomorphism $x \equiv x \otimes 1_{K}$. We define a conditional expectation $\mathbb{E}^{\mathcal{M} \otimes 1_{K}}$ by putting, for $y \in \mathcal{N}=\mathcal{M} \otimes B(K)$

$$
\mathbb{E}^{\mathcal{M} \otimes 1_{K}}(y)=\left(\Pi^{*} y \Pi\right) \otimes 1_{K},
$$

where $\Pi \xi=\xi \otimes \eta_{1}, \xi \in H$. It is easy to check that $\mathbb{E}^{\mathcal{M} \otimes 1_{K}}$ is a projection of norm one, so conditional expectation [7, p. 116]. Taking $\Phi$ as in Proposition 3.4, we have $\alpha x=\Pi^{*} \Phi(x) \Pi$, so

$$
\alpha x \otimes 1_{K}=\left(\Pi^{*} \Phi(x) \Pi\right) \otimes 1_{K}=\mathbb{E}^{\mathcal{M} \otimes 1_{K}} \Phi(x),
$$

which is equivalent to $(3.7)$.

Now, keeping notation as in Section 2, our Theorem 2.1 can be rewritten in the following way:

TheOREM 3.6. For a $W^{*}$-algebra $\mathcal{M}$ and for a measure $Q: \Sigma \rightarrow C P(\mathcal{M})$, there exists a $W^{*}$-algebra $\mathcal{N}, \mathcal{N} \supset \mathcal{M}$ (i.e. $\mathcal{M}$ is a $W^{*}$-subalgebra of $\left.\mathcal{N}\right)$ and a spectral measure $e: \Sigma \rightarrow \operatorname{Proj} \mathcal{N}$ such that

$$
Q(\Delta) x=\mathbb{E}^{\mathcal{M}}(e(\Delta) \Phi(x))
$$

for some *-representation $\Phi$ of $\mathcal{M}$ in $\mathcal{N}$ and a conditional expectation $\mathbb{E}^{\mathcal{M}}$ of $\mathcal{N}$ onto $\mathcal{M}$.

4. Dilations in conditional expectations scheme. In this section we compare our results of Sections 2 and 3 with theorems concerning measures with values being positive operators in $L_{1}$. It turns out that these results can be reformulated to the case of the algebra $L_{\infty}$ and then treated as theorems on commutative $W^{*}$-algebras.

In this context, constructiong a dilation, we shall try to use most natural transformations (projections) appearing in the $L_{1}$-space theory, like conditional expectation, indicator multiplication operator etc.

Moreover, we use a conditional expectation $E_{P}^{\mathcal{A}}$ for some probability measure $P$ (and $\sigma$-field $\mathcal{A}$ ) instead of a projection $P_{H}: \mathcal{H} \rightarrow H$ (from beyond the Hilbert space $H$ ).

Using here the space $L_{1}$ instead of $L_{\infty}$ seems to be a better idea.

Let $(X, \Sigma)$ be a topological Borel measurable space. Let $(M, \mathfrak{M}, \mu)$ be a probability space. A map $Q: \Sigma \rightarrow B\left(L_{1}(M, \mathfrak{M}, \mu)\right)$ is said to be a regular positive operator measure (shortly PO-measure) if the following conditions are satisfied:

1. $Q(\Delta) f \geq 0$ for $0 \leq f \in L_{1}$;

2. $Q\left(\bigcup_{s=1}^{\infty} \Delta_{s}\right) f=\sum_{s=1}^{\infty} Q\left(\Delta_{s}\right) f$, for $f \in L_{1}$, and pairwise disjoint $\Delta_{i}$ 's, the series being convergent in $L_{1}(M, \mathfrak{M}, \mu)$;

3. $Q$ is regular in the sense that for each $\varepsilon>0$ and each $\Delta \in \Sigma$ there exist in $X$ a compact set $Z$ and an open set $V$ such that

$$
\int_{M} Q(V-Z) 1_{M} d \mu<\varepsilon, \quad Z \subset \Delta \subset V ;
$$


4. $Q(X) 1_{M} \leq 1_{M}$;

5. $\int_{M} Q(X) f d \mu \leq \int_{M} f d \mu, \quad 0 \leq f \in L_{1}$.

We have the following

THEOREM 4.1 [4]. Let $Q$ be a regular positive operator measure. Then there exist a 'huge' measure space $(\Omega, \mathcal{F}, P)$, a $\sigma$-field $\mathcal{A} \subset \mathcal{F}$, a $\sigma$-lattice homomorphism $e: \Sigma \rightarrow \mathcal{F}$ and two measurable maps $i: \Omega \stackrel{\text { onto }}{\longrightarrow} M, j: \Omega \stackrel{\text { onto }}{\longrightarrow} M$ such that

$$
(Q(\Delta) f) \circ j=\mathbb{E}_{P}^{\mathcal{A}} 1_{e(\Delta)}(f \circ i), \quad \Delta \in \Sigma, \quad f \in L_{1}(M) .
$$

ThEOREM 4.2 [4]. There exist a measurable space $(\Omega, \mathcal{F})$, a measurable map $i: \Omega \rightarrow$ $M$ (onto), $\sigma$-fields $\mathcal{A}, \mathcal{B} \subset \mathcal{F}$, a $\sigma$-lattice homomorphism $e: \Sigma \rightarrow \mathcal{F}$, a set $\Omega_{0} \in \mathcal{F}$ such that, for every PO-measure $Q: \Sigma \rightarrow B\left(L_{1}(M, \mathfrak{M}, \mu)\right)$, there exists a probability measure $P$ on $(\Omega, \mathcal{F})$, for which the following formula holds:

$$
(Q(\Delta) f) \circ i=4 \mathbb{E}_{P}^{\mathcal{A}} 1_{e(\Delta)} \mathbb{E}_{P}^{\mathcal{B}} 1_{\Omega_{0}}(f \circ i), \quad \Delta \in \Sigma, \quad f \in L_{1}(M)
$$

For other similar results we refer to [4].

\section{References}

[1] L. Accardi and M. Ohya, Compound channels, transition expectations and liftings, preprint.

[2] O. Bratteli and D. W. Robinson, Operator algebras and quantum statistical mechanics, I, II New York-Heidelberg-Berlin, Springer, (1979).

[3] D. E. Evans and J. T. Lewis, Dilation of irreversible evolutions in algebraic quantum theory, Communications of the Dublin Institute for Advanced Studies, Series A (Theoretical Physics) 24 (1977).

[4] E. Hensz-Chądzyńska, R. Jajte and A. Paszkiewicz, Dilation theorems for positive operator-valued measures, Probab. Math. Statist. 17 (1997), 365-375.

[5] K. R. Parthasarathy, A continuous time version of Stinespring's theorem on completely positive maps, Quantum probability and Applications V, Proceedings, Heidelberg 1988, L. Accardi, W. von Waldenfels (eds.), Lecture Notes Math., Springer-Verlag (1988).

[6] W. F. Stinespring, Positive functions on $C^{*}$-algebras, Proc. Amer. Math. Soc. 6 (1965), 211-216.

[7] S. Strătilă, Modular theory in operator algebras, Editura Academiei, Bucuresti, Abacus Press (1981).

[8] S. Strătilă and L. Zsidó, Lectures on von Neumann algebras, Editura Academiei, Bucuresti, (1979).

[9] B. Sz.-Nagy, Extensions of linear transformations in Hilbert space which extend beyond this space, Appendix to: F. Riesz and B. Sz.-Nagy, Functional Analysis, Frederick Ungar Publishing Co.

[10] M. Takesaki, Theory of operator algebras, I, Springer, Berlin-Heidelberg-New York (1979). 